\title{
A COUNTEREXAMPLE TO A CONJECTURE IN LINEAR SECOND-ORDER DIFFERENTIAL EQUATIONS
}

\author{
KÁLMÁN SZABÓ
}

\begin{abstract}
The aim of the present paper is to give a simple counterexample to a conjecture [3] in linear second-order differential equations.
\end{abstract}

1. Introduction. Consider the following differential equation

$$
u^{\prime \prime}+a(t) \cdot u=0
$$

where $a(t)$ is a nondecreasing, positive and unbounded function in $C^{\prime}[T, \infty)$. It is well known that the hypotheses on $a(t)$ do not imply that every solution of (1) satisfies the condition

$$
u(t) \rightarrow 0 \text { as } t \rightarrow \infty \text {. }
$$

L. A. Gusarov [2] has shown that under the additional hypothesis that $a^{\prime}(t)$ is of bounded variation on $[T, \infty)$, the solutions of (1) satisfy condition (2). Under these assumptions, $a^{\prime}(t)$ has a finite, nonnegative limit as $t \rightarrow \infty$. A. Meir, D. Willett and J. S. W. Wong [3] have proved the following theorem.

THEOREM 1. If there exists a positive function $p(t) \in C^{\prime}[0, \infty)$ such that

$$
\int_{0}^{\infty} \frac{d t}{p(t)}=+\infty, \liminf _{t \rightarrow \infty} \frac{p^{\prime}(t)}{p(t) \cdot a^{1 / 2}(t)} \geq 0 \text { and } \liminf _{t \rightarrow \infty} \frac{a^{\prime}(t) \cdot p(t)}{a(t)}>0,
$$

then the solutions of (1) satisfy condition (2).

From this theorem it follows that if $a^{\prime}(t)$ is ultimately bounded and bounded away from zero, then all solutions of (1) satisfy (2). The following question presents itself: does the condition that $a^{\prime}(t) \rightarrow 0$ as $t \rightarrow \infty$ (or that $\lim \sup a^{\prime}(t)<\infty$ ) imply that condition (2) holds for all solutions of (1)?

Meir, Willett and Wong [3] conjectured that if in Theorem 1 the last condition is replaced by the condition

$$
\lim _{t \rightarrow \infty} a^{\prime}(t) \cdot p(t) / a(t)=0,
$$

then the conclusion remains valid. If this conjecture were true, we could answer our question in the affirmative (simply set $p(t) \equiv 1$ ). However, the following theorem shows that the conjecture is false.

Received by the editors November 3, 1981.

1980 Mathematics Subject Classification. Primary 34A30; Secondary 34B05.

Key words and phrases. Linear second-order differential equation, function of bounded variation. 
2. The counterexample. We can summarize our results as follows:

THEOREM 2. For each $\alpha>0$, there exists a positive function $a(t) \in C^{\infty}[0, \infty)$ such that $a(t) \rightarrow \infty, a^{\prime}(t) \geq 0, a^{\prime}(t)=o\left(\log ^{-\alpha} t\right)$ and such that at least one solution $u(t)$ of (1) satisfies the condition $\lim \sup _{t \rightarrow \infty}|u(t)|>0$.

Without loss of generality, we replace the condition $a^{\prime}(t)=o\left(\log ^{-\alpha} t\right)$ by $a^{\prime}(t)=$ $O\left(\log ^{-l} t\right)$, where $l$ is an integer $(l>\alpha)$.

The proof of Theorem 2 is based on a method used by A. S. Galbraith, E. J. McShane and G. B. Parrish [1], and D. Willett [5]. The following lemma, which was established by Willett [5], will be used in the proof of Theorem 2 .

LEMMA. Let $u(t)$ be a solution of (1), and let $\mu$ be a positive number such that $a(t) \geq \mu^{2}$ for all $t \in[0, \infty)$. Then $u^{\prime}(t)$ has at least one zero in each interval of length $2 \pi / \mu$.

Proof of Theorem 2. Consider the functions $A(t)$ and $B(t)$ defined by

$$
A(t)=\left\{\begin{array}{ll}
\exp \left(1-1 / t^{2}\right) & \text { for } t>0, \\
0 & \text { for } t \leq 0,
\end{array} \quad B(t)=A[1-A(1-t)] .\right.
$$

Clearly, $B(t)$ is a nondecreasing $C^{\infty}$-function with values in $[0,1]$, and it satisfies the conditions $B(t)=0, t \leq 0$, and $B(t)=1, t \geq 1$.

Let $t_{1}=0, q_{1}=1 / 2, \beta_{0}=16 \pi^{2}$ and $\beta_{1}=\beta_{0}+1$. Define $a_{1}(t)$ by condition $a_{1}(t)=\beta_{0}+\left(\beta_{1}-\beta_{0}\right) \cdot B(2 t)$. Let $u_{1}$ denote the unique solution of the initial value problem

$$
u_{1}^{\prime \prime}+a_{1}(t) \cdot u_{1}=0, \quad u_{1}(0)=1 \text { and } u_{1}^{\prime}(0)=0 .
$$

By the Lemma, there exists a point $t_{2}\left(1 / 2 \leq t_{2}<1\right)$ such that $u_{1}^{\prime}\left(t_{2}\right)=0$.

The following construction is inductive. We choose a sequence $\left\{\beta_{n}\right\}$, a sequence $0=t_{1}<q_{1}<t_{2}<q_{2}<\cdots$, and a set of functions $u_{n}(t)(n=1,2, \ldots)$ such that

$$
\begin{gathered}
q_{n}-t_{n}= \begin{cases}\min \left[n^{-1} \cdot \log ^{l} n\right], & \text { if } n \geq 2, \\
1 / 2, & \text { if } n=1,\end{cases} \\
n-3 / 2 \leq t_{n} \leq n-1, \\
\beta_{n}=\frac{1}{n}+\beta_{n-1}=\sum_{k=1}^{n} \frac{1}{k}+16 \pi^{2}, \\
a_{n}(t)=\beta_{n-1}+\left(\beta_{n}-\beta_{n-1}\right) \cdot B\left(\left(t-t_{n}\right) /\left(q_{n}-t_{n}\right)\right), \\
u_{n}^{\prime \prime}+a_{n}(t) \cdot u_{n}=0, \quad u_{n}\left(t_{n}\right)=u_{n-1}\left(t_{n}\right), \quad u_{n}^{\prime}\left(t_{n}\right)=u_{n-1}^{\prime}\left(t_{n}\right)=0 .
\end{gathered}
$$

Letting $\chi\left[t_{n}, t_{n+1}\right)$ denote the characteristic function of the half-open interval, we set

$$
a(t)=\sum_{n=1}^{\infty} a_{n}(t) \cdot \chi\left[t_{n}, t_{n+1}\right) \text { and } u(t)=\sum_{n=1}^{\infty} u_{n}(t) \cdot \chi\left[t_{n}, t_{n+1}\right) .
$$

We see that $a(t)$ is a positive, nondecreasing function belonging to $C^{\infty}[0, \infty)$, and that $u(t)$ satisfies the differential equation (1). Since $\beta_{n} \rightarrow \infty$ as $n \rightarrow \infty$, it follows that $a(t) \rightarrow \infty$ as $t \rightarrow \infty$. 
We now establish a bound on $a^{\prime}(t)$. Differentiating $a(t)$, we obtain the equation

$$
a^{\prime}(t)= \begin{cases}\frac{1}{q_{n}-t_{n}} \cdot B^{\prime}\left(\frac{t-t_{n}}{q_{n}-t_{n}}\right) \cdot\left(\beta_{n}-\beta_{n-1}\right) & \text { for } t_{n} \leq t \leq q_{n}, \\ 0 & \text { for } q_{n} \leq t \leq t_{n+1} .\end{cases}
$$

Since $B(t)$ is a $C^{\infty}$-function having compact support, $B^{\prime}(t)$ is bounded by some positive number $M$. Hence, for $t_{n} \leq t \leq t_{n+1}$ and $n \geq 2$ :

$$
a^{\prime}(t) \leq \frac{1}{q_{n}-t_{n}} \cdot\left(\beta_{n}-\beta_{n-1}\right) \cdot M<2 \cdot M \cdot \log ^{-l} n .
$$

For $t_{n} \leq t \leq t_{n+1}$ and $n \geq 5$, it follows from the condition $n-3 / 2 \leq t_{n} \leq n-1$ that $\log n \geq \log t_{n+1} \geq \log t \geq 1$. Combining this with (3), we obtain the estimate $a^{\prime}(t)=O\left(\log ^{-l} t\right)$.

To show that limsup $\sup _{t \rightarrow \infty}|u(t)|>0$, we choose numbers

$$
\varphi_{n}=1 / 2 \cdot\left(q_{n}-t_{n}\right)^{2} \cdot a\left(q_{n}\right) \quad(n \geq 2) .
$$

Since $\lim _{t \rightarrow \infty}\left(\log ^{k} t\right) \cdot t^{-1 / 2}=0$ for each positive integer $k$, there exists an integer $N$ such that

$$
16 \pi^{2}\left(\log ^{2 l} n\right) \cdot n^{-1 / 2} \leq 1 \text { and } 2 \cdot\left(\log ^{2 l+1} n\right) \cdot n^{-1 / 2} \leq 1,
$$

whenever $n \geq N$. Since each $\varphi_{n}(n \geq N)$ satisfies the inequalities

$$
\begin{aligned}
\varphi_{n} & =\frac{1}{2}\left[\sum_{k=1}^{n} \frac{1}{k}+16 \pi^{2}\right] \cdot n^{-2} \cdot \log ^{2 l} n \leq \frac{1}{2}\left[n^{-3 / 2}+(1+\log n) n^{-2} \cdot \log ^{2 l} n\right] \\
& \leq 2^{-1} \cdot\left[n^{-3 / 2}+2 \cdot n^{-2} \cdot \log ^{2 l+1} n\right] \leq n^{-3 / 2}
\end{aligned}
$$

we see that $\sum_{n=1}^{\infty} \varphi_{n} \leq \sum_{n=1}^{\infty}\left(1 / n^{3 / 2}\right)<\infty$.

We now show that

$$
\left|u\left(t_{n}\right)\right| \cdot\left[1-\varphi_{n}\right] \leq\left|u\left(t_{n+1}\right)\right|
$$

for each of the points $t_{n}$. By Taylor's theorem, we set

$$
t_{n} \leq c \leq q_{n} ; \quad u\left(q_{n}\right)=\left(q_{n}-t_{n}\right)^{2} \cdot u^{\prime \prime}(c) / 2 !+u\left(t_{n}\right) .
$$

We note that $\left|u^{\prime \prime}(c)\right|=a(c) \cdot|u(c)|$ and that $a(c) \leq a\left(q_{n}\right)$. It is well known [4, Part 2 , p. 28] that the values $\left|u\left(\eta_{i}\right)\right|$ determined by the points $\eta_{i}(i=1,2, \ldots)$ where $u^{\prime}\left(\eta_{i}\right)=0$ form a decreasing sequence. Therefore, $|u(c)| \leq\left|u\left(t_{n}\right)\right|$. From these observations we obtain the relations

$$
\begin{aligned}
\left|u\left(q_{n}\right)\right| & =\left|u\left(t_{n}\right)+\left(q_{n}-t_{n}\right)^{2} \cdot u^{\prime \prime}(c) / 2 !\right| \\
& \geq\left[1-\frac{1}{2}\left(q_{n}-t_{n}\right)^{2} \cdot a\left(q_{n}\right)\right] \cdot\left|u\left(t_{n}\right)\right|=\left[1-\varphi_{n}\right] \cdot\left|u\left(t_{n}\right)\right| .
\end{aligned}
$$

To estimate $\left|u\left(t_{n+1}\right)\right|$, we integrate the expression

$$
u^{\prime \prime} \cdot u^{\prime}+a \cdot u^{\prime} \cdot u=0
$$

by parts and obtain the equation

$$
a\left(t_{n+1}\right) \cdot u^{2}\left(t_{n+1}\right)=\left(u^{\prime}\left(q_{n}\right)\right)^{2}+a\left(q_{n}\right) \cdot u^{2}\left(q_{n}\right)+\int_{q_{n}}^{t_{n+1}} u^{2}(t) \cdot a^{\prime}(t) d t
$$


From this we deduce that

$$
u^{2}\left(t_{n+1}\right) \geq u^{2}\left(q_{n}\right) \cdot a\left(q_{n}\right) \cdot a^{-1}\left(t_{n+1}\right)=u^{2}\left(q_{n}\right) .
$$

Combining (5) with this inequality, we obtain (4).

Since $\sum_{n=1}^{\infty} \varphi_{n}<\infty$, there exists a positive integer $N$ such that $0<\varphi_{n}<1$ for $n \geq N$. From inequality (4), we see that

$$
\left|u\left(t_{N}\right)\right| \cdot \prod_{k=N}^{n}\left(1-\varphi_{k}\right) \leq\left|u\left(t_{n+1}\right)\right| .
$$

Since the product $\prod_{k=N}^{\infty}\left(1-\varphi_{k}\right)$ converges to some positive number, we deduce that limsup $\operatorname{su}_{t \rightarrow \infty}|u(t)|>0$, and this completes the proof.

ACKNOWLEDGEMENT. I am thankful to the referee for helpful comments.

\section{REFERENCES}

1. A. S. Galbraith, E. J. McShane and G. B. Parrish, On the solutions of linear second-order differential equations, Proc. Nat. Acad. Sci. U.S.A. 53 (1965), 247-249.

2. L. A. Gusarov, On the approach to zero of the solutions of a linear differential equation of the second order, Dokl. Akad. Nauk SSSR (N.S.) 71 (1950), 9-12 (Russian); reviewed in Math. Rev. 11 (1950), 516.

3. A. Meir, D. Willett and J. S. W. Wong, On the asymptotic behavior of the solutions of $x^{\prime \prime}+$ $a(t) x=0$, Michigan Math. J. 14 (1967), 47-52.

4. G. Sansone, Equazioni differenziali nel campo reale, Nicola Zanichelli, Bologna, 1948.

5. D. Willett, On an example in second order linear ordinary differential equations, Proc. Amer. Math. Soc. 17 (1966), 1263-1266.

Department of MAThematics, Kossuth UNIVERSity, Debrecen, HuNgary 